OUTP-98-32P

hep-th/9805027

September 1998

\title{
Black String Entropy from Anomalous D-brane Couplings
}

\author{
David D. Song" And Richard J. Szabo" \\ Department of Physics \\ University of Oxford \\ Oxford OX1 3PU, U.K.
}

\begin{abstract}
The quantum corrections to the counting of statistical entropy for the $5+1$ dimensional extremal black string in type-IIB supergravity with two observers are studied using anomalous Wess-Zumino actions for the corresponding intersecting D-brane description. The electric-magnetic duality symmetry of the anomalous theory implies a new symmetry between D-string and D-fivebrane sources and renders opposite sign for the RR charge of one of the intersecting D-branes relative to that of the black string. The electric-magnetic symmetric Hilbert space decomposes into subspaces associated with interior and exterior regions and it is shown that, for an outside observer, the expectation value of a horizon area operator agrees with the deviation of the classical horizon area in going from extremal to near-extremal black strings.
\end{abstract}

\footnotetext{
${ }^{1}$ E-mail: d.song1@physics.oxford.ac.uk

${ }^{2}$ Work supported in part by PPARC (U.K.); Address after September 1, 1998: The Niels Bohr Institute, University of Copenhagen, Blegdamsvej 17, DK-2100 Copenhagen $\varnothing$, Denmark

E-mail: szabo@alf.nbi.dk
} 
A startling consequence of the D-brane description of solitonic states [1] is the ability to microscopically count the statistical entropy of black holes in string theory. By realizing that intersecting D-branes are the weak coupling limit of certain five-dimensional supergravity black hole solutions and carry the same Ramond-Ramond (RR) charge as that of the black hole, the counting of quantum states from BPS-saturated D-brane bound states can be shown to agree with the Bekenstein-Hawking formula (proportional to the horizon area) for the entropy [2, 3] (see [4 for a review). In this letter we shall study quantum corrections to a class of classical black hole solutions in the D-brane description by considering the points of view of two observers where one is the observer outside the horizon and the other is a "superobserver" who, as advocated in the proposal of black hole complementarity [5], sees both exterior and interior regions of black holes (i.e. the Hilbert space of both exterior and interior regions is relevant). A natural way to understand the black hole/D-brane correspondence is to identify a quantum area operator on D-brane Hilbert space whose classical limit yields the horizon area of the extremal black hole in $N=8$ supergravity [6]. The five-dimensional black hole can be viewed as a six-dimensional black string which winds around a compact internal circle. The horizon area of the extremal black string and the expectation value of the area operator agree only in the limit of large winding number [6]. In an effort to find the location of the quantum states responsible for entropy counting, it was shown in [7] that the D-strings and D-fivebranes carrying the same RR charges as the black strings can be placed at the singularity of the interior region of the black string solution and argued that their modes stretch from the singularity out to the horizon.

When D-branes intersect on a topologically non-trivial manifold, the twistings of their normal bundles yield chiral anomalies in their worldvolume field theories which induce anomalous couplings of the D-brane gauge and gravitational fields to the RR tensor potentials [8]-[10]. The induced Wess-Zumino terms imply that topological defects (such as instantons or monopoles) on the D-branes carry their own RR charge determined by their topological quantum numbers [1]. In the following we will show that, when one carefully takes into account of the anomalies which occur on intersecting D-branes, while the RR charge of one D-brane coincides with the RR charge of black string, the RR charge of the other intersecting D-brane is of opposite sign.

Following [7], we interpret this effect as placing, at strong coupling, one D-brane in the interior and the other in the exterior region of the black string horizon. This change arises from the fact that the anomalous Wess-Zumino terms should be properly understood [9] as part of a total action which treats electric and magnetic potentials and sources on equal footing. This electric-magnetic symmetry decomposes the total Hilbert space symmetrically in terms of the individual D-brane Hilbert spaces. This leads to an intriguing symmetry between interchange of the two intersecting D-branes and hence of the interior and exterior regions of the black hole. We shall argue that this new

\footnotetext{
${ }^{3}$ This symmetry is not to be confused with the S-duality symmetry of type-IIB superstring theory.
} 
interpretation leads to the explanation of the discrepancy raised in the black hole/D-brane correspondence [6]. Indeed, this new picture leads us to consider both superobserver's and outside observer's points of view and we will show that, for an outsider observer, this interpretation effectively perturbs the classical extremal black string solution to the near-extremal case. The horizon area from this modified black string solution agrees with the expectation value of the area operator on D-brane Hilbert space. Thus the proper incorporation of anomalous D-brane couplings sheds new light on the role of observers in the quantum D-brane picture.

We start by briefly reviewing some properties of six-dimensional black strings in type IIB string theory. The bosonic action for type IIB supergravity in the Einstein frame is of the form

$$
\frac{1}{16 \pi G_{10}} \int_{\mathcal{M}_{10}} d^{10} x \sqrt{-g}\left(R-\frac{1}{2}(\nabla \phi)^{2}-\frac{1}{12} e^{\phi} H_{(3)}^{2}\right)+\ldots
$$

where the dots denote non-covariant field terms, $H_{(3)}$ is the RR three-form field strength, $\phi$ is the dilaton field, and $G_{10}$ is the ten-dimensional gravitational constant which in string units is given by $G_{10}=8 \pi^{6} g_{s}^{2}$ with $g_{s}$ the string coupling constant. The black string solution is most easily constructed on the ten-dimensional spacetime $\mathcal{M}_{10}=T^{5} \times R^{5}$ with the five-torus $T^{5}$ taken to be the product of a circle $S^{1}$ (along which the string lies), with coordinate $z$ and circumference $L$, and a four-torus $T^{4}$ with coordinates $y^{i}$ and hypervolume $V$. The Cartesian coordinates of the remaining $4+1$ asymptotically flat non-compact directions are denoted by $\left(x^{i}, t\right)$. In the limit $L \gg V^{1 / 4}$ the extremal black string solution in $5+1$ dimensions reduces to the $4+1$ dimensional Reissner-Nordström black hole of type IIB superstring theory.

In light-cone coordinates $u \equiv t-z, v \equiv t+z$, the classical six-dimensional exterior and interior black string solutions of the coupled Einstein equations which follow from the action (11) are [7]

$$
\begin{aligned}
d s_{\mathrm{ext}}^{2} & =h_{1}^{1 / 4} h_{5}^{3 / 4}\left[\frac{d u}{h_{1} h_{5}}(-d v+K d u)+\frac{d y_{i} d y^{i}}{h_{5}}+d x_{i} d x^{i}\right] \\
d s_{\text {int }}^{2} & =h_{1}^{1 / 4} h_{5}^{3 / 4}\left[\frac{d u}{h_{1} h_{5}}(d v+K d u)+\frac{d y_{i} d y^{i}}{h_{5}}+d x_{i} d x^{i}\right] \\
e^{-2 \phi} & =\frac{h_{5}}{h_{1}} \\
H_{(3) \nu u v} & =h_{1}^{-2} \partial_{\nu} h_{1} \quad, \quad H_{(3) i j k}=-\epsilon_{i j k l} \partial_{x_{l}} h_{5}
\end{aligned}
$$

where the harmonic functions are

$$
h_{1}=1+\frac{r_{1}^{2}}{r^{2}} \quad, \quad h_{5}=1+\frac{r_{5}^{2}}{r^{2}}
$$

for the exterior metric (2) and

$$
h_{1}=-1+\frac{r_{1}^{2}}{r^{2}} \quad, \quad h_{5}=-1+\frac{r_{5}^{2}}{r^{2}}
$$


for the interior metric (3), with $r^{2}=x_{i} x^{i}$. The horizon lies at the coordinate singularity $r=0$ in (2, 3) and the timelike curvature singularity lies at either $r=r_{1}$ (if $r_{1}<r_{5}$ ) or $r=r_{5}$ (if $r_{5}<r_{1}$ ). The constants $r_{1}$ and $r_{5}$ determine the electric and magnetic charges of the black hole with respect to the RR three-form field strength by

$$
\begin{aligned}
Q_{1} & \equiv \frac{V}{(2 \pi)^{6} g_{s}} \int_{S^{3}} e^{\phi} *_{6} H_{(3)}=\frac{V r_{1}^{2}}{(2 \pi)^{4} g_{s}} \\
Q_{5} & \equiv \frac{1}{(2 \pi)^{2} g_{s}} \int_{S^{3}} H_{(3)}=\frac{r_{5}^{2}}{g_{s}}
\end{aligned}
$$

where $S^{3}$ is a three-sphere and $*_{6}$ the Hodge dual in the six-dimensional spacetime $R^{5} \times S^{1}$. With this normalization the charges $Q_{1}$ and $Q_{5}$ are integers. For simplicity, we will consider only the homogeneous case where $K=p / r^{2}$ with $p$ the momentum of the black hole. Note that, aside from the forms of the harmonic functions, the only difference between the exterior and interior solutions is the change in sign of the $d u d v$ term in the metric.

By putting a $u$ dependence on the momentum $p \equiv p(u)$, one can add travelling waves [12], i.e. waves moving along the string, since $p(u)$ then changes the momentum density along the string. For extremal black holes, there are only left-moving modes, and therefore $p$ depends on $u$ but not on $v$. The corresponding horizon area is

$$
A=2 \pi^{2} r_{1} r_{5} V \int \sqrt{p(u)} d u
$$

When $p \equiv(2 \pi)^{6} g_{s}^{2} N / L^{2} V$ is constant, with $N$ the number of modes, (10) yields the familiar extremal Bekenstein-Hawking entropy

$$
S_{e}=\frac{A}{4 G_{10}}=2 \pi \sqrt{Q_{1} Q_{5} N}
$$

of the $4+1$ dimensional black hole.

Being sources which carry RR charge, the electric and magnetic charges can be induced by superposing $Q_{1}$ D-string and $Q_{5}$ D-fivebrane sources with worldvolumes $M_{1}$ and $M_{5}$ lying in the $(t, z)$ plane $R^{1} \times S^{1}$ and the $5+1$ dimensional $\left(t, z, y^{i}\right)$ space $R^{1} \times S^{1} \times$ $T^{4}$, respectively. The D-strings and D-fivebranes intersect along the worldsheet of the string. Since the D-strings wind $Q_{1}$ times around the $S^{1}$ and the D-fivebranes wind $Q_{5}$ times around the whole $T^{5}$, the effective string wraps $Q_{1} Q_{5}$ times around the compact $z$ direction. This feature is the key to counting the entropy (11) using D-branes. Using the positive energy theorem for black holes, it is argued in [7] that outside the horizon, BPS D-brane solutions with positive charge and energy density are static whereas D-branes with negative charge and energy density are unphysical. Inside the horizon, the situation is opposite in that it is the negatively charged D-brane or anti-D-brane which is static (and has positive energy density).

The comparison of black hole states to quantum configurations of the D-branes can be made by constructing an area operator for the black string on the D-brane Hilbert space 
$\mathcal{H}_{D}$ [6]. This is achieved by defining a momentum operator and plugging it into (10). We will consider the case when $\sqrt{p} \ll r_{1}, r_{5}$. The ensemble of homogeneous black string states contains those states which lie on a single string of tension $1 / 2 \pi g_{s} Q_{5}$ and within a length scale $\tilde{L} \equiv Q_{1} Q_{5} L$. The string can be described by a free worldsheet $\sigma$-model for a single bosonic field $X$, which in the limit $L \gg V^{1 / 4}$ describes the excitations of the D-branes. Then the momentum density along the string can be written as $T_{++}=\left(\partial_{+} X\right)^{2} / 2 \pi g_{s} Q_{5}$, whose mode expansion is given by $\partial_{+} X=\left(\sqrt{2 \pi^{2} g_{s} Q_{5}} / \tilde{L}\right) \sum_{m=-\infty}^{\infty} \alpha_{m} e^{-2 \pi i m \sigma^{+} / \tilde{L}}$ with $\left[\alpha_{m}, \alpha_{n}\right]=m \delta_{-m, n}$. Since the differences between the worldsheet coordinate $\sigma^{+}$and the spacetime coordinate $u$ are integer multiples of $L$ due to the string wrapping around the compact direction $Q_{1} Q_{5}$ times, it follows that the parameters of the black string solution can be identified with quantum fields on the effective string worldsheet through the momentum operator

$$
p(u)=\frac{1}{\tilde{L}} \sum_{l=-\infty}^{\infty} p_{l} e^{-2 \pi i l u / \tilde{L}}
$$

where

$$
p_{l}=\frac{2 \pi^{2} g_{s}^{2}}{V \tilde{L}} \sum_{k=1}^{Q_{1} Q_{5}} \sum_{n=-\infty}^{\infty} e^{-2 \pi i k l L / \tilde{L}}: \alpha_{l-n} \alpha_{n}:
$$

Note that in (13) the index $k$ runs from 1 to $Q_{1} Q_{5}$.

Using the equipartition theorem, it can be shown [6] that the difference between the expectation value of the area operator $(\sqrt{10})$ in $\mathcal{H}_{D}$ and the area of the classical stationary black string can be estimated by evaluating the quantity $\sum_{k \neq 0} \frac{<: p_{k} p_{-k}:>}{<: p_{0}^{2}:>}$. The quantum fluctuations in the longitudinal momentum yield the deviation

$$
\sum_{k} \frac{<: p_{k} p_{-k}:>}{<: p_{0}^{2}:>} \sim \frac{1}{Q_{1} Q_{5}}
$$

from the area of the stationary black hole [6]. Therefore for large winding number $Q_{1} Q_{5}$, the two areas can match. But generally there is always a deviation between the classical horizon area and the expectation value of the area operator on $\mathcal{H}_{D}$ which leads to an entropy counting difference

$$
\frac{\Delta S}{S_{e}} \sim \frac{1}{\sqrt{Q_{1} Q_{5}}}
$$

between the classical and (quantum) D-brane approaches.

In the following we will describe an origin for the discrepancy (15). D-brane field theory plays a vital role in the counting of statistical entropy for a quantum description of black holes at strong coupling [2]- [4]. At low energy, these field theories have both gauge and global symmetries. Anomalies arise due to the chiral asymmetry, with respect to the global R symmetry, of massless Weyl fermion fields on the intersection of D-branes. These anomalies can be compensated for by the anomalous variation of the classical D-brane actions. In [8]-10] it was shown that the classical variations of Wess-Zumino actions for the D-branes cancel the Yang-Mills and gravitational anomalies as well as the anomalies associated with global R symmetries. Let us now quickly describe how this works. 
The low-energy dynamics of the configuration of $Q_{1}$ D-strings and $Q_{5}$ D-fivebranes, each with infinitesimal separation, are described, respectively, by supersymmetric field theories on the worldvolumes $M_{1}$ and $M_{5}$ which have $U\left(Q_{1}\right)$ and $U\left(Q_{5}\right)$ gauge symmetries [1, 13]. The anomalous gauge variation on the intersection of D-strings and D-fivebranes on the effective string worldsheet $M_{1} \cap M_{5}$ can be written as [8]-10]

$$
2 \pi \int_{\mathcal{M}_{10}} \triangle_{M_{1}} \wedge \triangle_{M_{5}} \wedge\left(\operatorname{ch}\left(F_{1}\right) \wedge \operatorname{ch}\left(-F_{5}\right) \wedge \frac{\hat{A}\left[T\left(M_{1}\right) \cap T\left(M_{5}\right)\right]}{\hat{A}\left[N\left(M_{1}\right) \cap N\left(M_{5}\right)\right]}\right)^{(1)}
$$

where the delta-function supported Poincaré dual form $\triangle_{M_{i}}$, of degree $10-\operatorname{dim} M_{i}$, is the de Rham current of $M_{i}$, i.e. $\int_{M_{i}} Z \equiv \int_{\mathcal{M}_{10}} \triangle_{M_{i}} \wedge Z$. If $N$ is the leading constant part of the closed gauge-invariant form $Z \equiv N+d Z^{(0)}$, then $Z^{(1)}$ denotes its corresponding Wess-Zumino descendent defined by the first-order gauge variation $\delta_{g} Z^{(0)} \equiv d Z^{(1)}$ of its secondary characteristic. The tangent bundle $T\left(\mathcal{M}_{10}\right)$ of the total space decomposes locally as $T\left(M_{i}\right) \oplus N\left(M_{i}\right)$, with $N\left(M_{i}\right)$ the normal bundle of the embedded submanifold $M_{i}$, and $\hat{A}$ denotes the Dirac genus. $F_{1}$ and $F_{5}$ are Hermitian $U\left(Q_{1}\right)$ and $U\left(Q_{5}\right)$ gauge field strengths, respectively, and

$$
\operatorname{ch}\left(F_{j}\right) \equiv \operatorname{tr}_{Q_{j}} \exp \frac{i F_{j}}{2 \pi}=Q_{j}+\operatorname{ch}_{1}\left(F_{j}\right)+\operatorname{ch}_{2}\left(F_{j}\right)+\ldots
$$

are the corresponding Chern characteristic classes in the fundamental $Q_{j}$ representations of the respective gauge groups on the D-branes. The anomaly term (16) comes from the tensor product of the spinor bundles, associated with the lifting of $T\left(\mathcal{M}_{10}\right)=$ $T\left(M_{i}\right) \oplus N\left(M_{i}\right)$, with the Chan-Paton vector bundles over the D-branes. As shown in [8], the anomalous zero modes on the intersection of the D-branes come from the massless excitation spectrum of the D-brane field theory which consists of Weyl fermions in the mixed sector $Q_{1} \otimes \bar{Q}_{5}$ and $\bar{Q}_{1} \otimes Q_{5}$ representations of the gauge group $U\left(Q_{1}\right) \times U\left(Q_{5}\right)$. Since the Chern characteristic class for the adjoint representation of the unitary group can be decomposed as $\operatorname{ch}\left[U\left(Q_{i}\right)_{Q_{i} \otimes \bar{Q}_{i}}\right]=\operatorname{ch}\left(F_{i}\right) \wedge \operatorname{ch}\left(F_{i}^{*}\right)=\operatorname{ch}\left(F_{i}\right) \wedge \operatorname{ch}\left(-F_{i}\right)$, the $\operatorname{ch}\left(F_{1}\right) \wedge \operatorname{ch}\left(-F_{5}\right)$ term comes from the mixed sectors $Q_{1} \otimes \bar{Q}_{5}$ and $\bar{Q}_{1} \otimes Q_{5}$ which both contribute equally.

The anomaly term (16) can be cancelled by carefully writing the constituent bulk D-brane actions in well-defined forms. The Wess-Zumino part of the low-energy effective action for the D-brane dynamics has the form

$$
I_{\mathrm{WZ}}=-\frac{\mu}{2} \sum_{i=1,5} \int_{M_{i}} C \wedge Z_{i}
$$

where $\mu$ is the $\mathrm{D}$-brane charge, $C$ is the sum of all the even form-degree $\mathrm{RR}$ gauge fields for the type-IIB theory we are considering here, and $Z_{i}$ are the D-brane sources which are invariant polynomials of the Yang-Mills field strengths and gravitational curvatures on $M_{i}$. Denoting the $p$-form parts of $C$ and $Z_{i}$ by $C_{(p)}$ and $Z_{i(p)}$, the action (18) can be written as an integral over the total spacetime using the de Rham currents as

$$
\begin{aligned}
I_{\mathrm{WZ}}=-\frac{\mu}{2} \int_{\mathcal{M}_{10}} & \left\{\triangle_{M_{1}} \wedge\left(C_{(0)} \wedge Z_{1(2)}+C_{(2)} \wedge Z_{1(0)}\right)\right. \\
+ & \left.\triangle_{M_{5}} \wedge\left(C_{(0)} \wedge Z_{5(6)}+C_{(2)} \wedge Z_{5(4)}+C_{(4)} \wedge Z_{5(2)}+C_{(6)} \wedge Z_{5(0)}\right)\right\}
\end{aligned}
$$


where we recall that $\triangle_{M_{1}}$ is an eight-form and $\triangle_{M_{5}}$ is a four-form.

However, because of the D-brane couplings, the gauge fields are not globally-defined as single-valued differential forms, and (18) should be written more carefully since $H$, the total RR field strength, has global corrections to its local form $d C$. The appropriate modification is the anomalous D-brane coupling

$$
I_{\mathrm{WZ}}=-\frac{\mu}{2} \sum_{i=1,5} \int_{\mathcal{M}_{10}} \triangle_{M_{i}} \wedge\left(N_{i} C-H \wedge Z_{i}^{(0)}\right)
$$

which coincides with (18) upon integration by parts when $H=d C$, but otherwise contains global corrections to the form (18). The equations of motion which follow from coupling the kinetic term of (四) to the source action (20) are

$$
d\left(e^{\phi} *_{10} H\right)=\mu \sum_{i=1,5} \triangle_{M_{i}} \wedge Z_{i}
$$

where $*_{10}$ is the Hodge dual in the ten-dimensional spacetime $\mathcal{M}_{10}$, and the Bianchi identities are 9

$$
d H=-\mu \sum_{i=1,5} \triangle_{M_{i}} \wedge \tilde{Z}_{i}
$$

where $\tilde{Z}_{i(l)}=(-1)^{\left(\operatorname{dim} M_{i}+l+2\right) / 2} Z_{i(l)}$ is the conjugate to $Z_{i}$ defined by reversing the orientations of the Chan-Paton and tangent bundles over the D-branes. The gauge variation of the potential is thus $\delta_{g} C=\mu \sum_{i} \triangle_{M_{i}} \wedge \tilde{Z}_{i}^{(1)}$, and it follows that the gauge variation of the modified Wess-Zumino action (20) is

$$
\delta_{g} I_{\mathrm{WZ}}=-\frac{\mu^{2}}{2} \int_{\mathcal{M}_{10}} \triangle_{M_{1}} \wedge \triangle_{M_{5}} \wedge\left(Z_{1} \wedge \tilde{Z}_{5}+Z_{5} \wedge \tilde{Z}_{1}\right)^{(1)}
$$

Therefore with

$$
\begin{aligned}
& Z_{i}=\operatorname{ch}\left(F_{i}\right) \wedge \sqrt{\frac{\hat{A}\left[T\left(M_{i}\right)\right]}{\hat{A}\left[N\left(M_{i}\right)\right]}} \\
& \tilde{Z}_{j}=\operatorname{ch}\left(-F_{j}\right) \wedge \sqrt{\frac{\hat{A}\left[T\left(M_{j}\right)\right]}{\hat{A}\left[N\left(M_{j}\right)\right]}}
\end{aligned}
$$

and $\mu^{2}=2 \pi$, the variation (23) cancels the anomaly term (16).

The Bianchi identity (22) follows from the fact that the anomalous coupling (20) explicitly involves both electric and magnetic sources and is to be understood as part of an action which is manifestly electric-magnetic symmetric [9]. As we will now demonstrate, the anomalous action (20) with this underlying electric-magnetic duality yields new implications for the quantum descriptions of black holes in the D-brane picture. We note first of all that the field strength $H$ in $(21,22)$ is the sum of all the odd form-degree RR field strengths for the type-IIB theory. To compare the physical D-brane charges derived from the anomalous coupling (20) with those of the black string solution of (1), we are interested in the three-form component $H_{(3)}$ of $H$. We therefore consider two hypervolumes 
$B^{8}$ and $B^{4}$ in $\mathcal{M}_{10}$ whose boundaries are spheres $S^{7}$ and $S^{3}$ enclosing the total D-string and D-fivebrane charge. From (21) and (22) we havef

$$
\begin{aligned}
\int_{B^{8}} d\left(e^{\phi} *_{10} H_{(3)}\right) & =\mu \int_{B^{8}}\left(\triangle_{M_{1}} \wedge Z_{1(0)}+\triangle_{M_{5}} \wedge Z_{5(4)}\right) \\
\int_{B^{4}} d H_{(3)} & =-\mu \int_{B^{4}} \triangle_{M_{5}} \wedge \tilde{Z}_{5(0)}
\end{aligned}
$$

The first term on the right-hand side of (26) corresponds to the physical charge $\mathcal{Q}_{1}$ of the D-strings, while the second term is an induced anomaly charge. This latter charge arises from the fact [11] that RR charge conservation requires the boundaries of the D-string to carry instanton number with respect to the D-fivebrane worldvolume gauge field. The right-hand side of (27) represents the physical charge $\mathcal{Q}_{5}$ of the D-fivebranes.

For the D-string and D-fivebrane worldvolumes we have $\tilde{Z}_{i(0)}=Z_{i(0)}$. It follows from (24), (17) and the fact that the constant part of the Dirac genus is 1 that

$$
\begin{aligned}
& \mathcal{Q}_{1}=\mu Q_{1} \\
& \mathcal{Q}_{5}=-\mu Q_{5}
\end{aligned}
$$

We see that, for $\mu=+\sqrt{2 \pi}$, the RR charge of D-strings coincides with (8) while the RR charge of D-fivebranes is of opposite sign to (9). .] This would seem to contradict that D-branes, at weak coupling, carry the same RR charge as that of the black hole.

The crucial minus sign which appears in (29) comes from the electric-magnetic duality property of the anomalous Wess-Zumino coupling (20). The total (non-covariant) explicitly duality symmetric action $I_{B E}+I_{\mathrm{WZ}}$ comes from writing the field strength term in the supergravity action (1) as [9, 14]

$$
I_{B E}=-\frac{1}{384 \pi G_{10}} \int_{\mathcal{M}_{10}} e^{\phi}\left(B_{(3)} \wedge E_{(7)}+B_{(7)} \wedge E_{(3)}+B_{(3)} \wedge *_{10} B_{(3)}+B_{(7)} \wedge *_{10} B_{(7)}\right)
$$

where we have decomposed $H_{(p)}=E_{(p)}+B_{(p)}$ for $p=3,7$ in terms of its components $E_{(p)}$ with a temporal index and its components $B_{(p)}$ involving only spatial indices on $\mathcal{M}_{10}$. Thus when one interprets the D-string and D-fivebrane fields as ten-dimensional electromagnetic duals of one another, the physical couplings of the black string change. This symmetry is necessary to cancel the anomalous fermionic zero modes (16) via a change of orientation of the bundles over one of the intersecting D-branes.

Generally, the construction of explicitly electric-magnetic symmetric quantum field theories involves augmenting the Hilbert space so as to include two independent gauge potentials where one is the physical field and the other is the unphysical dual field [14, 15]. In the present case the two potentials are associated each with the D-string and Dfivebrane in $\mathcal{M}_{10}$, and corresponding sources $Z_{i}$ and $\tilde{Z}_{i}$, which in turn means that the full

\footnotetext{
${ }^{4}$ Note that here, in contrast to the RR charges (8) and (9) which are defined in the six-dimensional black string frame, we equivalently define D-brane charge in a ten-dimensional frame.

${ }^{5}$ For $\mu=-\sqrt{2 \pi}$, RR charge of D-strings would have opposite sign to (8)
} 
D-brane Hilbert space to be considered is the tensor product

$$
\mathcal{H}_{D}=\mathcal{H}_{D 1} \otimes \mathcal{H}_{D 5}
$$

of independent D-string and D-fivebrane Hilbert spaces. Since the Hilbert space of the exterior and interior regions of the black hole is relevant only to a superobserver, one could associate the unphysical dual field with the interior region of the black hole for an outside observer, i.e. one of the intersecting D-branes is associated with exterior while the other is associated with interior region of black string. It was argued that [7] in placing Dbranes in static equilibrium on the extremal black hole, outside the horizon the positively charged D-brane is static whereas inside the horizon the negatively charged D-brane is static. Therefore, if we consider that, at strong coupling and for $\mu=+\sqrt{2 \pi}$ (which we henceforth assume), the D-string is placed in the exterior while the D-fivebrane should be interpreted as lying on the interior of the black string horizon, then this resolves the sign difference between the RR charges (29) and (9).

The decomposition of the Hilbert space (31) can then also be thought of as yielding Hilbert spaces $\mathcal{H}_{D 1}$ for the exterior and $\mathcal{H}_{D 5}$ for the interior regions of the quantum black hole. This augmented Hilbert space of exterior and interior regions can be of use only to a superobserver [5]. From a superobserver's point of view, both the D-string and D-fivebrane are positively charged and only left moving momentum modes exist. This augmented Hilbert space also gives a remarkable manifestation of the electric-magnetic symmetry as an interior-exterior region symmetry of the black string. The horizon, where the D-branes intersect, then corresponds to projecting the Hilbert space (31) onto only one component representing the physical (duality non-symmetric) solution in which both D-branes are placed in the same region. With this projection onto "physically observable" states (i.e. those relevant to an outside observer), the RR three-form field strength and its dual are related by $H_{(3)}=-*_{10} H_{(7)}$ [9], which gives $E_{(7)}=-*_{10} B_{(3)}$ and $E_{(3)}=-*_{10} B_{(7)}$ in (30) leaving only one set of electromagnetic tensor fields for the physical black string solution.

This interpretation also modifies the momentum operator (12) on the D-brane Hilbert space, which with respect to the decomposition (31) can be considered as a function $p\left(u_{1}, u_{5}\right)$ of two variables $u_{1}$ and $u_{5}$ representing the worldsheet coordinates of the Dstring and D-fivebrane, respectively. The corresponding mode expansion (12, 13) can be rewritten as

$$
p\left(u_{1}, u_{5}\right)=\frac{1}{\tilde{L}} \sum_{l_{1}, l_{5}=-\infty}^{\infty}\left(p_{l_{1}} \otimes p_{l_{5}}\right) e^{-2 \pi i\left(l_{1} u_{1}+l_{5} u_{5}\right) / \tilde{L}}
$$

where

$$
\begin{aligned}
& p_{l_{1}}=\sqrt{\frac{2 \pi^{2} g_{s}^{2}}{V \tilde{L}}} \sum_{k_{1}=1}^{Q_{1}} \sum_{n_{1}=-\infty}^{\infty} e^{-2 \pi i k_{1} l_{1} L / \tilde{L}}: \alpha_{l_{1}-n_{1}}^{(1)} \alpha_{n_{1}}^{(1)}: \\
& p_{l_{5}}=\sqrt{\frac{2 \pi^{2} g_{s}^{2}}{V \tilde{L}}} \sum_{k_{5}=1}^{Q_{5}} \sum_{n_{5}=-\infty}^{\infty} e^{-2 \pi i k_{5} l_{5} L / \tilde{L}}: \alpha_{l_{5}-n_{5}}^{(5)} \alpha_{n_{5}}^{(5)}:
\end{aligned}
$$


and the modes $\alpha_{n_{1}}^{(1)}$ and $\alpha_{n_{5}}^{(5)}$ act on the Hilbert spaces $\mathcal{H}_{D 1}$ and $\mathcal{H}_{D 5}$, respectively. Note that the index $k_{1}$ runs from 1 to $Q_{1}$ for $p_{l_{1}}$ while $k_{5}$ runs from 1 to $Q_{5}$ for $p_{l_{5}}$. The modification (32,33) of (12, 13) does not change the deviation (14).

Unlike a superobserver, an outside observer, where only the exterior metric is relevant, sees branes and anti-branes. Moreover, the momentum $p_{l_{5}}$ corresponding to $Q_{5}$ is no longer left moving if the D-fivebrane intersects as an anti-brane. Instead it is right moving, i.e. $u_{5} \equiv v$. From the outside observer's point of view, the classical six-dimensional black string solution, with a new interpretation of the momentum operator in (32,33), i.e. with the D-fivebrane as an anti-brane and with right as well as left moving momentum, represents the non-extremal case. [5 The outside observer therefore sees the extremal horizon as if it were a non-extremal one. The corresponding Bekenstein-Hawking entropy is [3, 16]

$$
S_{n-e}=2 \pi \sqrt{Q_{1} Q_{5}}\left(\sqrt{N_{1}}+\sqrt{N_{5}}\right)
$$

where $N_{1,5}$ is the number of left,right moving modes. In the absence of right moving modes, (34) becomes the entropy (11) of the extremal black string. If the number of right movers is small, then the perturbation away from a purely left-moving extremal background is small. When a small number $\delta N_{5}$ of right moving oscillations is added, the change in entropy from the extremal case is

$$
\frac{\Delta S}{S_{e}}=\sqrt{\frac{\delta N_{5}}{N}}
$$

where $N \equiv N_{1}-N_{5}$. In terms of the mass change $\delta M$ due to the transition to nearextremality, we have [4]

$$
\frac{\Delta S}{S_{e}} \sim \sqrt{\frac{\delta M}{M_{e}}}
$$

where $M_{e}$ is the mass of the extremal black string.

The mass gap between the near-extremal and the extremal black string is [4]

$$
\frac{(\Delta p)^{2}}{M_{e}} \equiv \delta M \sim \frac{1}{Q_{1} Q_{5}}
$$

The entropy difference (36) thus coincides with the deviation (15) caused by the quantum fluctuations of the momentum of the stationary black string. As pointed out before, the decomposition (32) of the momentum operator into two independent components does not change the result (14). It is the area from the classical black string solution that is modified so as to include a right moving momentum, yielding an identical entropy deviation in both the classical and D-brane pictures. Therefore the discrepancy in the black hole/D-brane

\footnotetext{
${ }^{6}$ Although we have only considered left and right moving momentum modes and argued that (32) corresponds to the non-extremal case, it seems that the BPS supersymmetric properties of the configuration are also different for a superobserver and an outside observer. We leave the detailed study of BPS bound state configurations for these two observers for future research.
} 
correspondence can be explained from the fact that, unlike a superobserver, the outside observer does not have access to the interior of black holes. In other words, the discrepancy results from the projection of the augmented electric-magnetic symmetric Hilbert space onto physically observable states.

It would be interesting to see how the above interpretation of the D-brane Hilbert space based on anomalous couplings and electric-magnetic symmetry can be used to describe other issues in black hole physics. For instance, in [17] it is argued that pure quantum states do not form black holes, which suggests the need for quantum decoherence in the black hole description. What we have shown above suggests that there are interactions between the interior and exterior regions of a black hole at the horizon. Therefore it seems plausible to interpret the interior of a black hole as the environment or external

source in quantum decoherence. The quantum corrections, due to the external sources, to the entropy of four-dimensional Einstein-Yang-Mills black holes are described in [18]. Furthermore, the decomposition (31) of the Hilbert space of the quantum field theory into two independent components is the natural setting for the noncommutative geometry description of duality symmetries [15]. The approach above may therefore also be relevant to the noncommutative short-distance structure, which is inherent in D-brane field theory [13], of black hole spacetimes.

\section{References}

[1] J. Polchinski, Phys. Rev. Lett. 75 (1995) 4724; J. Polchinski, S. Chaudhuri and C. Johnson, hep-th/9602052

[2] A. Strominger and C. Vafa, Phys. Lett. B379 (1996) 99

[3] S.R. Das and S.D. Mathur, Phys. Lett. B375 (1996) 103; C.G. Callan and J.M. Maldacena, Nucl. Phys. B472 (1996) 591; G.T. Horowitz and A. Strominger, Phys. Rev. Lett. 77 (1996) 2368

[4] J.M. Maldacena, hep-th/9607235

[5] L. Susskind, L. Thorlacius and J. Uglum, Phys. Rev. D48 (1993) 3743

[6] D. Marolf, Phys. Rev. D57 (1998) 2427

[7] G.T. Horowitz and D. Marolf, Phys. Rev. D55 (1997) 3654

[8] M.B. Green, J.A. Harvey and G. Moore, Class. Quant. Grav. 14 (1997) 47

[9] Y.-K.E. Cheung and Z. Yin, Nucl. Phys. B517 (1998) 69

[10] R. Minasian and G. Moore, J. High Energy Phys. 11 (1997) 002 
[11] E. Witten, Nucl. Phys. B460 (1996) 541; M.R. Douglas, hep-th/9512077

[12] G.T. Horowitz and D. Marolf, Phys. Rev. D55 (1997) 846

[13] E. Witten, Nucl. Phys. B460 (1996) 335

[14] J.H. Schwarz and A. Sen, Nucl. Phys. B411 (1994) 5; P. Pasti, D. Sorokin and M. Tonin, Phys. Rev. D52 (1995) 4277; S. Deser, A. Gomberoff, M. Henneaux and C. Teitelboim, Phys. Lett. B400 (1997) 80

[15] F. Lizzi and R.J. Szabo, Phys. Lett. B417 (1998) 303

[16] G.T. Horowitz, J.M. Maldacena and A. Strominger, Phys. Lett. B383 (1996) 151

[17] R.C. Myers, Gen. Rel. Grav. 29 (1997) 1217

[18] N.E. Mavromatos and E. Winstanley, Phys. Rev. D53 (1996) 3190; J. Ellis, N.E. Mavromatos, D.V. Nanopoulos and E. Winstanley, Mod. Phys. Lett. A12 (1997) 243 